\title{
The performance of the Mocean M100 wave energy converter described through numerical and physical modelling
}

\author{
J. Cameron McNatt, and Christopher H. Retzler
}

\begin{abstract}
Mocean Energy has designed a $100-\mathrm{kW}$ hinged-raft wave energy converter (WEC), the M100, which has a novel geometry that reduces the cost of energy by improving the ratios of power per size and power per torque.

The performance of the M100 is shown through the outputs of frequency-domain and time-domain numerical models, which are compared with those from $1 / 20^{\text {th }}$ scale wave-tank testing.

Results show that for the undamped, frequency-domain model, there are resonant peaks in the response at 6.6 and 9.6 $\mathrm{s}$, corresponding to wavelengths that are 1.9 and 3.7 times longer than the machine. With the inclusion of power-take-off and viscous damping, the power response as a function of frequency shows a broad bandwidth and a hinge flex amplitude of 12-20 degrees per meter of wave amplitude.

Comparison between the time-domain model and physical data in a variety of sea states, up to a significant wave height of $4.5 \mathrm{~m}$, show agreements within $10 \%$ for average power absorption, which is notable because only simple, nonlinear, numerical models were used.

The M100 geometry results in a broad-banded, largeamplitude response due to its asymmetric shape, which induces coupling between modes of motion.
\end{abstract}

Keywords-wave energy converter, numerical modelling, tank testing, resonance.

\section{INTRODUCTION}

$\mathrm{T}^{\circ}$ make wave energy commercially viable, innovations are needed in main wave energy converter (WEC) subsystems such as the prime mover, power take off (PTO), structure, control system, and moorings and foundations. The need for system wide innovation, particularly following the folding of several prominent wave energy companies in 2014-15, has driven the formation of innovative funding programs, such as

Manuscript received 16 March; accepted 25 March; published 12 May, 2020. This is an open access article distributed under the terms of the Creative Commons Attribution 4.0 licence (CC BY http://creativecommons.org/licenses/by/4.0/). This article has been subject to single-blind peer review by a minimum of two reviewers. This report is work commissioned by Wave Energy Scotland. The views expressed in this publication are those of the author(s) and not necessarily those of Wave Energy Scotland. J. C. McNatt and C. Retzler are and the inventors of the M100WEC the co-founders of Mocean Energy Ltd, Edinburgh, Scotland (website: www.moceanenergy.com; e-mail: cameron.mcnatt@moceanenergy.com, chris.retzler@moceanenergy.com). Digital Object Identifier https://doi.org/10.36688/imej.3.11-19

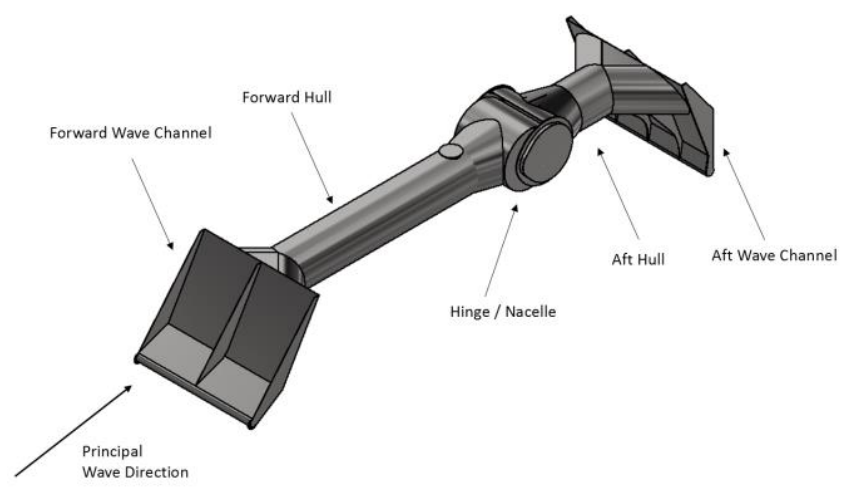

Fig. 1. The Mocean M100 WEC with labels indicating key geometric features. The figure shows the M100a; Mocean is currently developing an iteration beyond this that can be found at www.moceanenergy.com and is referred to as the M100b.

Wave Energy Scotland (WES) [1]. Through the WES Novel WEC program, Mocean Energy has developed its WEC from a simple geometric concept to a complete design for a 100-kW WEC referred to as the M100.

The M100, shown in Fig. 1, is a hinged raft: two hulls connected by a single revolute joint parallel to the wave crests. Wave forcing and the bodies' dynamic responses cause a relative motion about the hinge, which drives a PTO producing electricity.

Hinged-raft WECs date back to patents submitted in the late 1970s and early 1980s by Cockerell [2] and Hagen [3]. They are frequently referred to as attenuators (however, the authors contend that this term should only be used for rafts with multiple PTO joints positioned down the length of the machine).

Hinged rafts present several engineering benefits. Because energy is absorbed through the relative motion of one hull against the other and not by acting against a mooring, mooring loads are low, resulting in less expensive and simpler mooring systems. Another benefit is that the WEC system can be connected and disconnected from its mooring quickly, which reduces installation costs and allows it to be towed between installation site and port reducing maintenance costs and improving safety.

However, three major challenges exist for traditional hinged rafts:

1) The length of the machine must be approximately equal to that of the wavelengths from which energy 
TABLE I

DIMENSIONS OF THE M100

\begin{tabular}{lll}
\hline Measure & Units & Value \\
\hline Length overall & $\mathrm{m}$ & 36.7 \\
Length forward hulla $^{\mathrm{a}}$ & $\mathrm{m}$ & 24.8 \\
Length aft hull $^{\mathrm{a}}$ & $\mathrm{m}$ & 10.8 \\
Draft & $\mathrm{m}$ & 6.0 \\
Mass overall & $\mathrm{kg}$ & 261,480 \\
Mass forward hull & $\mathrm{kg}$ & 182,520 \\
Mass aft hull & $\mathrm{kg}$ & 78,960 \\
aThe hull lengths are given from the hinge to the furthest extent.
\end{tabular}

is absorbed, which can be from 70 to $140 \mathrm{~m}$ (representing 6.7 to $9.2 \mathrm{~s}$ waves in $50 \mathrm{~m}$ water depth).

2) Hinged rafts typically have small angular rotational responses to waves (less than $10^{\circ}$ arcs). As power for a hinged raft is the product of torque and hinge velocity, large torque levels are required, which leads to an expensive PTO system.

3) In extreme seas, waves can lift the forward WEC hull out of the water; it then slams upon re-entry, creating a problematic load case.

The M100 addresses these challenges through its novel geometry, which is characterized by asymmetry, and by features that Mocean refers to as "wave channels", found on both the forward and aft hulls. A wave channel is a sloped, mostly submerged plate with sidewalls, which may have an inflection into a plate at a different slope.

The hulls are asymmetric in length (the forward hull is longer than the aft), asymmetric in their wave channel shapes, and each hull is asymmetric along its length; each hull is shaped like an " $\mathrm{L}$ ".

The wave channels project down into the wave flow increasing the wave excitation forces and providing additional wave-making damping (which is needed for wave energy absorption); they entrap fluid creating beneficial added mass forces.

The geometry of the M100 was found with a genetic algorithm optimization, which used an underlying numerical model. Software was developed to programmatically create geometries based on a parametric definition, compute the hydrodynamic coefficients with WAMIT [4], and then compute annual energy production using the spectral method developed by Folly [5] to model the nonlinear viscous forces and nonlinear torque limit.

The impact of the geometry is that the dynamic response of the M100 has resonances at multiple wavelengths, which are longer than the length of the machine. This results in large motions and a broad bandwidth. The resonant response means that a smaller machine can be built addressing above-mentionedchallenge 1) and that less torque is required for the same amount of power, addressing challenge 2). Challenge 3 is overcome because the forward wave channel always stays submerged and its sloping face causes the WEC to dive through large waves, avoiding slamming.

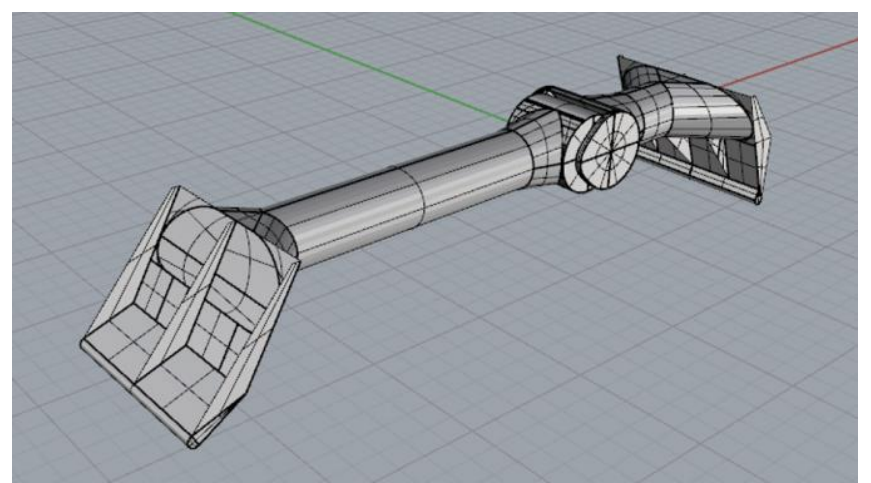

Fig. 3. The Mocean M100 WEC as a CAD model used to compute hydrodynamic coefficients in WAMIT.

Herein, numerical results are used to show the multiple resonant peaks and the broad-banded response. The numerical results are compared to wave tank testing results in both regular and irregular waves. The aim of this work is to present the M100 performance and behavior and elicit feedback from the scientific community.

\section{METHODS}

The performance and behavior of the M100 are evaluated using both a linear frequency domain model and a nonlinear time-domain model. Results from both are compared to those from a $1 / 20^{\text {th }}$ scale model tested in a wave tank.

\section{A. Numerical modelling}

The CAD geometry used for the numerical modeling is shown in Figure 3. It was implemented in Rhino3D as a series of untrimmed surfaces and exported as a WAMIT hi-order panel file. Thin surfaces were implemented in WAMIT as dipole panels.

Numerical modelling was carried out at full scale; fullscale masses are given in Table I. For the numerical modelling, masses, centers of gravity (CGs), and moments of inertia were measured from the small-scale physical model as built. The physical model had targets for mass and CG based on a concept design, but due the practicalities of building models, they could not be met; for example, the full-scale target mass was $220,000 \mathrm{~kg}$, however, the scaled mass of the physical model was $261,480 \mathrm{~kg}$.

The M100 hinge constraint reduces the degrees of freedom (DOF) from 12 (for two independent rigid bodies) to 7 DOF: surge, sway, heave, roll, pitch, yaw, and flex; flex is the relative pitch between the forward and aft hulls.

The constraint is implemented in both the frequencydomain and time-domain numerical models using a linear constraint matrix. For a force given in the unconstrained, 12-DOF system, $\boldsymbol{f}_{u}$, the force in the constrained, 7-DOF system is:

$$
\boldsymbol{f}_{c}=\boldsymbol{P} \boldsymbol{f}_{u}
$$


where $\boldsymbol{P}$ is the linear constraint matrix. Similarly, for a matrix in the unconstrained system, $\boldsymbol{G}_{u}$, the constrainedsystem matrix is:

$$
\boldsymbol{G}_{c}=\boldsymbol{P} \boldsymbol{G}_{u} \boldsymbol{P}^{T}
$$

Unconstrained body motions, $\boldsymbol{x}_{u}$, are found from constrained motions, $\boldsymbol{x}_{c}$, via the relationship:

$$
\boldsymbol{x}_{u}=\boldsymbol{P}^{T} \boldsymbol{x}_{c}
$$

The constraint matrix, $\boldsymbol{P}$, is found based on the relationship in position between the hinge and the origin of each hull (typically its CG) in a manner similar to that of [6] and [7].

The equations of motion for both the frequencydomain and the time-domain numerical models are given in the constrained DOF and the subscript, $c$, is omitted.

\section{1) Frequency-domain modelling}

The frequency-domain model solves the set of linear equations

$$
\begin{aligned}
{\left[-\omega^{2}(\boldsymbol{A}(\omega)+\boldsymbol{M})\right.} & +i \omega\left(\mathcal{B}(\omega)+\boldsymbol{D}_{P T O}+\boldsymbol{D}_{\boldsymbol{v}}\right) \\
+ & (\boldsymbol{K}+\boldsymbol{C})] \boldsymbol{\xi}(\omega, \beta) \\
= & a(\omega, \beta) \hat{\boldsymbol{f}}^{E}(\omega, \beta)
\end{aligned}
$$

for the complex motions amplitudes, $\xi(\omega, \beta)$, where $i=$ $\sqrt{-1}, \omega$ is the radial wave frequency, $\beta$ is the incident wave direction, and $\mathcal{A}(\omega)$ is the added mass matrix, $\boldsymbol{M}$ is the mass matrix, $\mathcal{B}(\omega)$ is the hydrodynamic damping matrix, $\boldsymbol{D}_{\text {PTO }}$ is the PTO damping matrix, $\boldsymbol{D}_{v}$ is a damping matrix that represents all viscous/frictional losses to the system, $\boldsymbol{K}$ is the mooring stiffness matrix, $\boldsymbol{C}$ is the hydrostatic stiffness matrix, $a(\omega, \beta)$ is a complex incident wave amplitude, $\hat{\boldsymbol{f}}^{E}(\omega, \beta)$ is the linear wave excitation force for a unit-amplitude wave.

The added mass, hydrodynamic damping, hydrostatic stiffness and wave excitation force are computed with WAMIT.

The PTO damping matrix consists of a real valued coefficient, $d_{P T O}$, in the flex-flex matrix position and zeros otherwise.

The viscous damping matrix, $\boldsymbol{D}_{\boldsymbol{v}}$, is developed by "slicing" geometry into so-called Morison elements and linearizing the nonlinear Morison force. The nonlinear Morison force on a given element in DOF $i$ (surge, sway, or heave) is

$$
f_{i}=-\frac{1}{2} c_{d} \rho A\left|v_{i}\right| v_{i}
$$

where $c_{d}$ is a nondimensional damping coefficient, $\rho$ is the fluid density, $A$ is the element area, and $v$ is he velocity of the element. This can be linearized using the Lorentz linearization [5] to be

$$
f_{i}=-\left[\frac{4}{3 \pi} c_{d} \rho A\right] v_{i}
$$

where the coefficient $\frac{4}{3 \pi}$ has units of velocity. For each 6 DOF rigid body, the terms in the brackets can be developed into a linear 6x6 linear viscous damping matrix which can be transformed into a constrained viscous damping matrix following equation 2 .

The mooring spring force matrix, $\boldsymbol{K}$, is formed by transforming the linear mooring force at a point on the body into a linear mooring stiffness matrix for a 6 DOF rigid body and then into constrained mooring stiffness matrix following equation 2 . At the point on the hull at which the mooring is connected, the linear mooring force in DOF $i$ is

$$
f_{i}=k_{i} x_{i}
$$

where $k_{i}$ is the mooring stiffness in DOF $i$ and $x_{i}$ is the displacement in DOF $i . k_{i}$ is found by summing the projected mooring stiffness of each of the mooring lines, where the stiffness of the mooring line was measured from the experimental system.

2) Time-domain modelling

The time-domain model solves the equation of motion

$$
\begin{gathered}
\left(\boldsymbol{A}_{\infty}+\boldsymbol{M}\right) \ddot{\boldsymbol{x}}+\int_{0}^{t} \boldsymbol{\kappa}(t-\tau) \dot{\boldsymbol{x}}(\tau) d \tau+(\boldsymbol{K}+\boldsymbol{C}) \boldsymbol{x} \\
=\boldsymbol{F}^{E}+\boldsymbol{F}^{V i s}+\boldsymbol{F}^{P T O}
\end{gathered}
$$

where $\boldsymbol{A}_{\infty}$ is the infinite frequency added mass matrix, $\boldsymbol{\kappa}$ is the radiation impulse response function, $\boldsymbol{F}^{E}$ is the wave excitation force, $\boldsymbol{F}^{V i s}$ is the viscous damping force, and $\boldsymbol{F}^{P T O}$ is the PTO damping force. In equation 8 , all terms are linear except $\boldsymbol{F}^{V i s}$ and $\boldsymbol{F}^{P T O}$.

The wave excitation force is

$$
\boldsymbol{F}^{E}=R(t) \cdot \operatorname{Re}\left\{\sum_{m=1}^{M} a\left(\omega_{m}, \beta_{m}\right) \hat{\boldsymbol{f}}^{E}\left(\omega_{m}, \beta_{m}\right) e^{i \omega_{m} t}\right\}
$$

where $R(t)$ is a ramp function used to begin a simulation.

The viscous force is found by summing the forces and moments due to all Morison elements using the nonlinear Morison forces given in equation 5.

The PTO force is zero in all DOF except flex. In the flex $\mathrm{DOF}$, the torque is linear and proportional to a linear damping coefficient, $\tau_{P T O}^{L}=-d_{P T O} v$, up to a torque limit, $\tau_{0}$, beyond which it is constant

$$
\tau_{P T O}=\left\{\begin{array}{cc}
-\tau_{0} & \tau_{P T O}^{L}<-\tau_{0} \\
\tau_{P T O}^{L} & -\tau_{0} \leq \tau_{P T O}^{L} \leq \tau_{0} \\
\tau_{0} & \tau_{P T O}^{L}>\tau_{0}
\end{array}\right.
$$




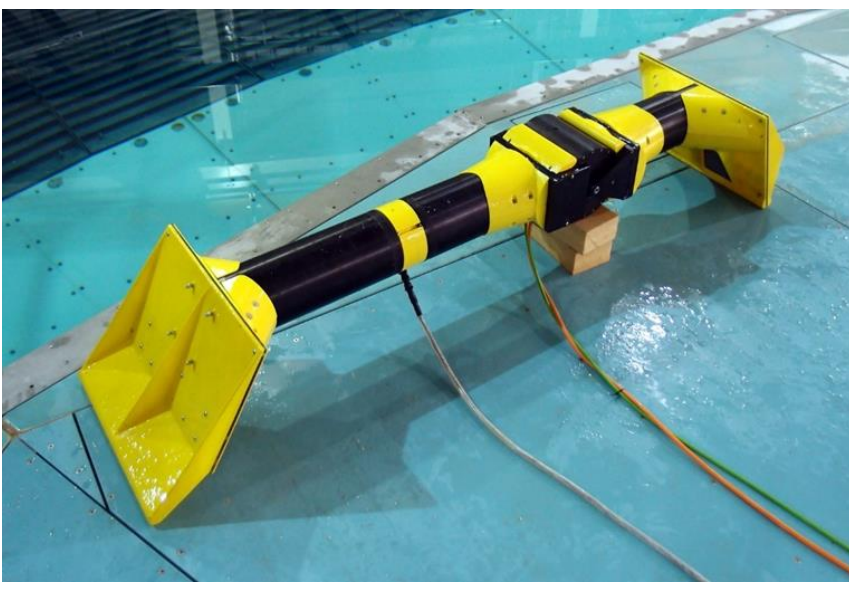

Fig. 4. The Mocean M100 WEC $1 / 20^{\text {th }}$ scale model.

\section{B. Physical modelling}

Mocean designed and built a $1 / 20^{\text {th }}$ scale model of the M100, shown in Fig 4, which was tested in the FloWave wave tank [8] in August 2018.

\section{1) Model description}

The model was assembled from machined and black anodized aluminium alloy and 3D printed parts in yellow ABS and PLA plastic. The plastic surfaces were sealed with an epoxy coating - although the sealing was not absolute and there was some water uptake.

The model was moored with a compliant 3-point spread mooring equispaced on a circle, with a forward mooring line aligned with the principal wave direction and two aft lines each at $60^{\circ}$ to the wave direction.

\section{2) Model PTO}

The model PTO consisted of a controllable motor in line with the hinge and mounted and sealed inside the nacelle. In Fig. 4, the green signal and orange power cable can be seen exiting from the nacelle. The motor incorporated a resolver that outputed the angular position and velocity of the shaft to 24-bit precision. A microprocessor controller provided demand signals to the motor via a current drive.

Via the controller a linear PTO damping value was set by providing a specific gain to the velocity dependent feedback loop. The nonlinear torque limit was set by setting a current limit to the motor.

\section{3) Instrumentation}

The model was instrumented with a variety of sensors. However, only the instrumentation used in the analysis presented here is discussed.

To measure the motions in 6 DOF of each hull, a Qualisys motion capture system was used.

Hinge velocity was measured using the motor encoder (but could also be derived from the Qualisys system).

Due to space constraints, it was not possible to include a torque sensor inline with the hinge-motor shaft. Instead, to measure torque, the motor current was used, where the current values were pre-calibrated to a torque value with

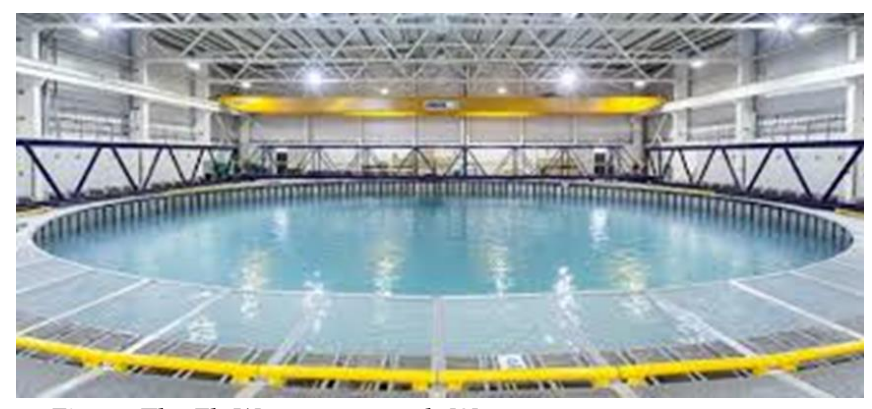

Fig. 5. The FloWave wave tank [8].

TABLE II

WAVE TANK TEST PERFORMED AND ANALYSED

\begin{tabular}{lllll}
\hline No. & Wave type & $\begin{array}{l}\text { Steepness } \\
(\lambda / H)\end{array}$ & $\begin{array}{l}\text { PTO } \\
\text { damping } \\
(\mathrm{MNms} / \mathrm{rad})\end{array}$ & $\begin{array}{l}\text { PTO torque } \\
\text { limit } \\
(\mathrm{MNm})\end{array}$ \\
\hline 1 & Regular & 100 & 6.1 & 1.50 \\
2 & Regular & 100 & 9.9 & 0.75 \\
3 & Regular & 50 & 9.9 & 1.50 \\
4 & Irregular & - & 9.9 & 1.00 \\
5 & Irregular & - & 6.1 & 1.00
\end{tabular}

an external torque sensor before the motor was fitted in the model.

\section{4) Wave tank}

The FloWave wave tank [8], shown in Figure 5, features a circular ring of wavemakers, $25 \mathrm{~m}$ in diameter. The tank floor can be raised to just above the water surface for model preparation. With the floor down, the water depth is $2 \mathrm{~m}$.

It can make waves of periods between 1 and $2.9 \mathrm{~s}$ and wave heights up to $0.4 \mathrm{~m}$. Using the scale of $1 / 20$, these correspond to periods of 4.5 to $13.0 \mathrm{~s}$, and a height of $8 \mathrm{~m}$.

\section{5) Tests performed}

Although more test sets than these were carried out over the course of the test program, 5 sets of tests are considered here, including both regular and irregular waves. These are summarized in Table II.

The regular waves were produced at two wave steepnesses, where the steepness is the ratio of the wavelength to the wave height: $\lambda / H$. 20 wave periods were covered ranging from 4.8 to $13.0 \mathrm{~s}$ at full scale. The periods were chosen so that an integer number of wave cycles occurred in the given test sample time to facilitate the Fourier transform.

12 sets of long-crested irregular waves were tested with energy periods $\left(T_{e}\right)$ ranging from 6.6 to $12.6 \mathrm{~s}$, and significant wave heights from 1.5 to $4.5 \mathrm{~m}$. Test -set No. 5 only used a subset of these wave conditions.

Two PTO damping values and three PTO torque limits were used, as given in Table II.

\section{Quantities used}

Numerical and physical test results are considered in terms of two quantities: 1) the magnitude of the sinusoidal body motion (for regular waves only), and 2) average power absorbed. 
The magnitude of the body motion is normalized by the incident wave amplitude as a quantity commonly referred to as the response amplitude operator (RAO). For the frequency-domain numerical model, the RAO is $|\xi / a|$. For the time-domain numerical model and for the wave tank tests, the magnitude of the motion is found as the magnitude of the lowest-order harmonic following the Fourier transform of the time-domain signal. In the wave tank, the magnitude of the incident wave was found from measurements of the waves in the tank without the model using a reflection wave gauge array.

Average power is the time-average of the product of the hinge torque and the hinge velocity

$$
\bar{P}=-\overline{\tau_{P T O} v_{h}}
$$

In the frequency-domain numerical model, this corresponds to

$$
\bar{P}=\frac{1}{2} \omega^{2} d_{P T O}\left|\xi_{P T O}\right|^{2}
$$

In regular waves, the average power is normalized by the incident amplitude squared to form the power RAO.

\section{RESULTS AND DISCUSSION}

For the M100 in regular head-on waves, there is no motion in sway, roll, or yaw, and the system can be reduced to 4 DOF: surge, heave, pitch and flex.

\section{Undamped response}

The undamped numerical response, Fig 6, shows the frequency-domain RAO for the numerical model in which the PTO damping, viscous damping, and mooring force are set to zeros. It is the response of the model as a mass-spring system due solely to linear inviscid hydrodynamics. Although not representative of reality, it is useful because it reveals information about the system's dynamic response.

In Fig 6., one can see that there are two clear resonant peaks a 6.6 and $9.6 \mathrm{~s}$, which appear in all DOFs, but most importantly in flex, the PTO DOF. Between the peaks, there is an extremely wide bandwidth response in flex between about $5 \mathrm{~s}$ and $12 \mathrm{~s}$. The saddle point between the peaks reaches a minimum RAO value of 28.1 degrees $/ \mathrm{m}$, which is very large.

The wavelengths of the resonant peaks of 6.6 and $9.6 \mathrm{~s}$ at $40 \mathrm{~m}$ water depth (the depth at which the calculations were performed) are 68 and $137 \mathrm{~m}$ respectively. These wavelengths are 1.9 and 3.7 times, respectively, longer than the device length. This means that a smaller, less expensive machine can be built to access longer, more energetic waves.

The reason that the M100 has two resonant frequencies in flex is due to coupling between modes of motion. Coupling is a force in one mode of motion due to motion
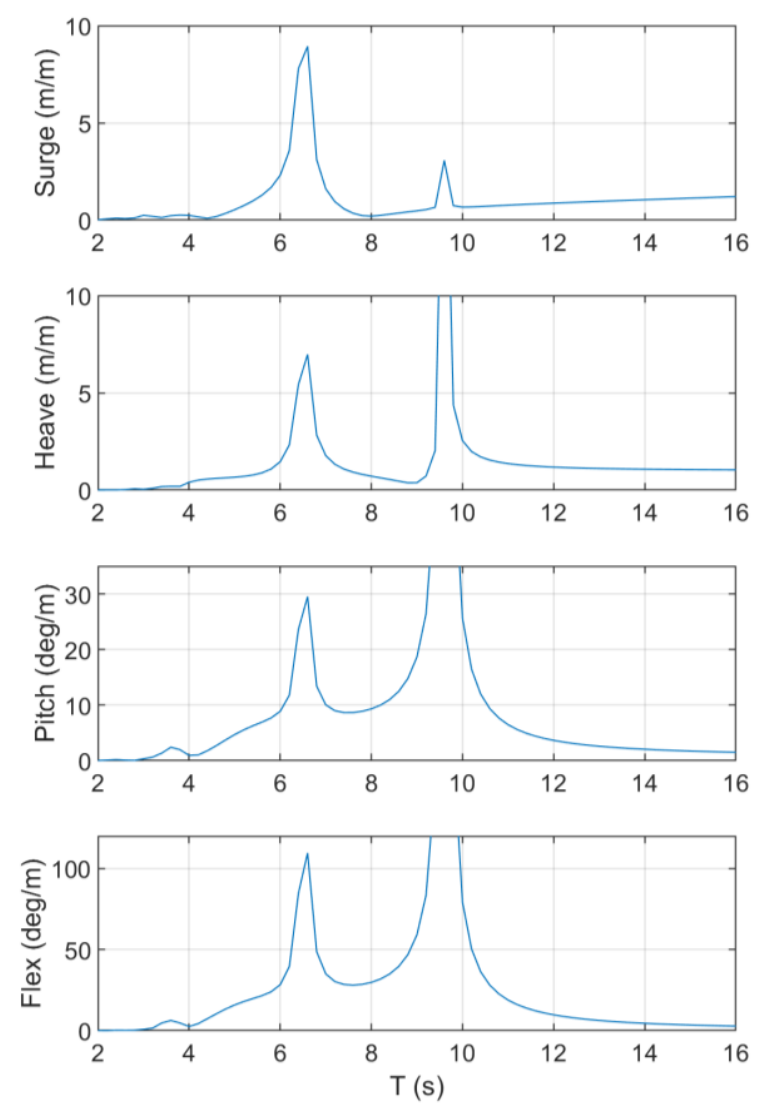

Fig. 6. Frequency-domain undamped response RAO.

in another. The coupling forces are represented by offdiagonal terms in the force matrix.

For a mass-spring system with $N$ DOF given by the equation

$$
M \ddot{x}+K x=0
$$

harmonic solutions are of the form

$$
\boldsymbol{K} \xi=\omega^{2} \boldsymbol{M} \xi
$$

where $\omega$ is a resonant frequency, $\omega^{2}$ is a generalized eigenvalue, and $\xi$ is so-called mode shape (initial position that causes resonance) and is also a generalized eigenvector. Such a system can have up to $N$ resonant frequencies.

However, if the matrices $\boldsymbol{M}$ and $\boldsymbol{K}$ are diagonal, then each eigenvector, $\xi$, will only have a single non-zero entry meaning that the response in each DOF is independent of other DOFs and that each DOF will only have a single resonant frequency.

In order to have multiple resonant frequencies in a single DOF, coupling between modes of motion, that is, off-diagonal terms in the mass/added mass matrix and stiffness matrices are required. The more off-diagonal terms are present, the more "flexibility" the system has to find solutions in which the resonance occurs at useful frequencies.

Because in the WEC frequency domain equation of motion, the "mass" is the sum of the mass matrix and the added mass terms, which are frequency dependent, and 


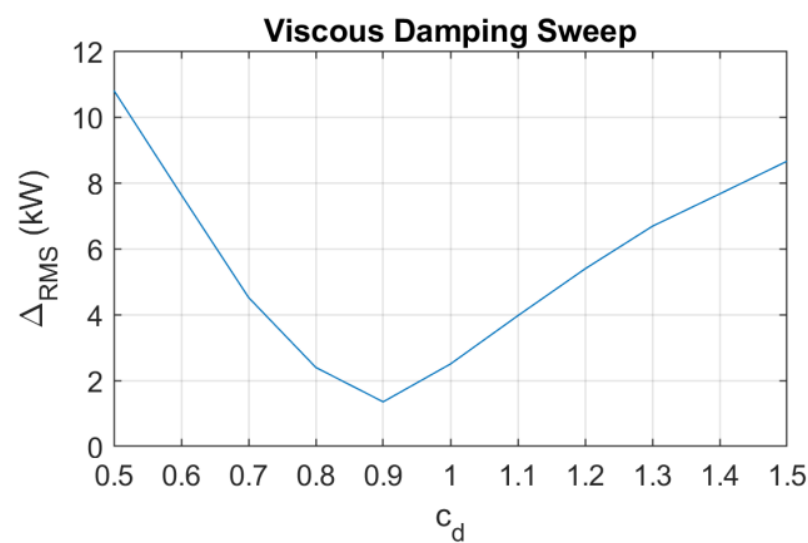

Fig. 7. Sweep of RMS difference between numerical and experimental power estimates for Test Set 4 as a function of $c_{d}$

because the frequency-dependent damping terms are significant, one cannot simple use equation 14 to find the resonant frequencies and eigenvectors. Instead it is more straightforward to solve equation 14 at each frequency and identify the resonant periods.

It can be shown that in contrast, the traditional symmetric hinged raft (each hull is the same length, and CG is at the hinge) has only an off-diagonal terms in heave-flex.

\section{E. Calibration}

With the exception of the nondimensional viscous damping coefficient, $c_{d}$, the coefficients used in the numerical results are computed either from the geometry using WAMIT or measured from the experimental set up.

However, $c_{d}$ is an empirical coefficient that depends on the nature of the geometry. In theory, there could be a different $c_{d}$ for each Morison element and in each DOF. However, for simplicity, only a single value of the viscous damping coefficient is used; this was found to be sufficient to produce a reasonable fit of model to data results.

The value used for $c_{d}$ is found by quantitatively comparing the numerical and the physical average power results over all tests in Test Set 4 (irregular waves). The comparison is made using the root-mean-square difference between the numerical, $\bar{P}_{n}$, and the physical average power, $\bar{P}_{p}$,

$$
\Delta_{R M S}=\sqrt{\frac{1}{N} \sum_{i=1}^{N}\left(\bar{P}_{n, i}-\bar{P}_{p, i}\right)^{2}}
$$

In the numerical model calibration, the value of $c_{d}$ is found that minimizes $\Delta_{R M S}$ by sweeping through a range of values of $c_{d}$ for the Test Set 4 (see Table II). Test Set 4 was selected because it was irregular waves, which are the conditions in which the WEC is meant to operate, and because it contained more runs than test set 5 . The numerical results use the time-domain model.

The results of the viscous damping sweep are given in Fig 7, from which one can see that the RMS difference is
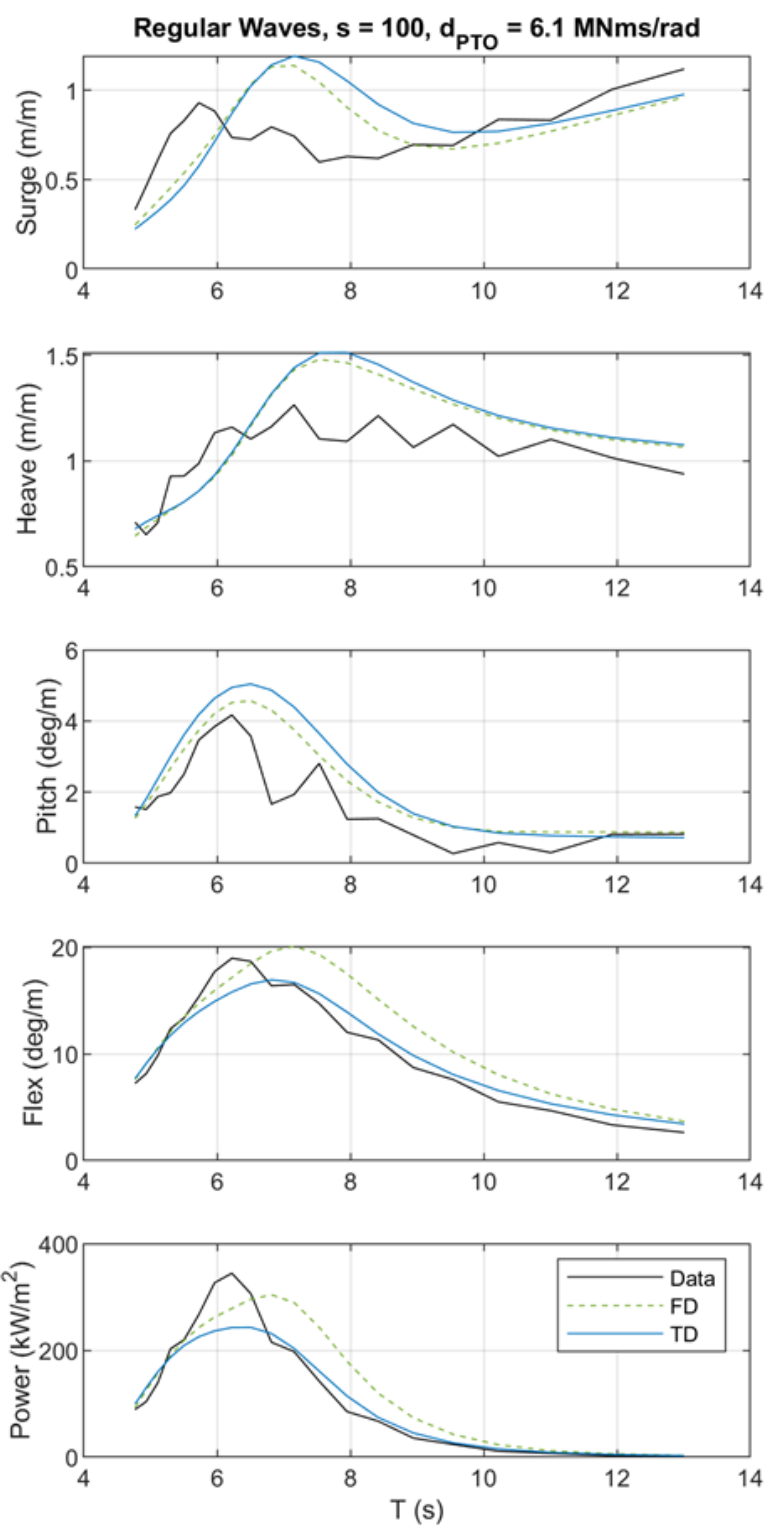

Fig. 8. RAO Test Set 1.

minimized for $c_{d}=0.9$. The value of $c_{d}=0.9$ is used for all subsequent results.

\section{F. Regular wave response}

Figures 8, 9, and 10 show the regular wave response for Test Cases 1, 2, and 3 respectively. Results are given RAOs for both the measured data (black solid line), the linear frequency-domain model (red dashed) and the nonlinear time-domain model (blue). Both motion RAOs in surge, heave, pitch and flex are shown as well as the power RAO.

There is reasonable agreement between the data and both numerical models in terms of the trends for pitch and flex for all Test Cases - peaks of the responses occur at the same periods and are of approximately the same magnitude.

It is also useful to compare the numerical and measure responses to that of the undamped frequency-domain model shown in Fig 6. The resonant peaks in flex shown in the undamped model are no longer present and the overall response is closer to the lower period resonance at 

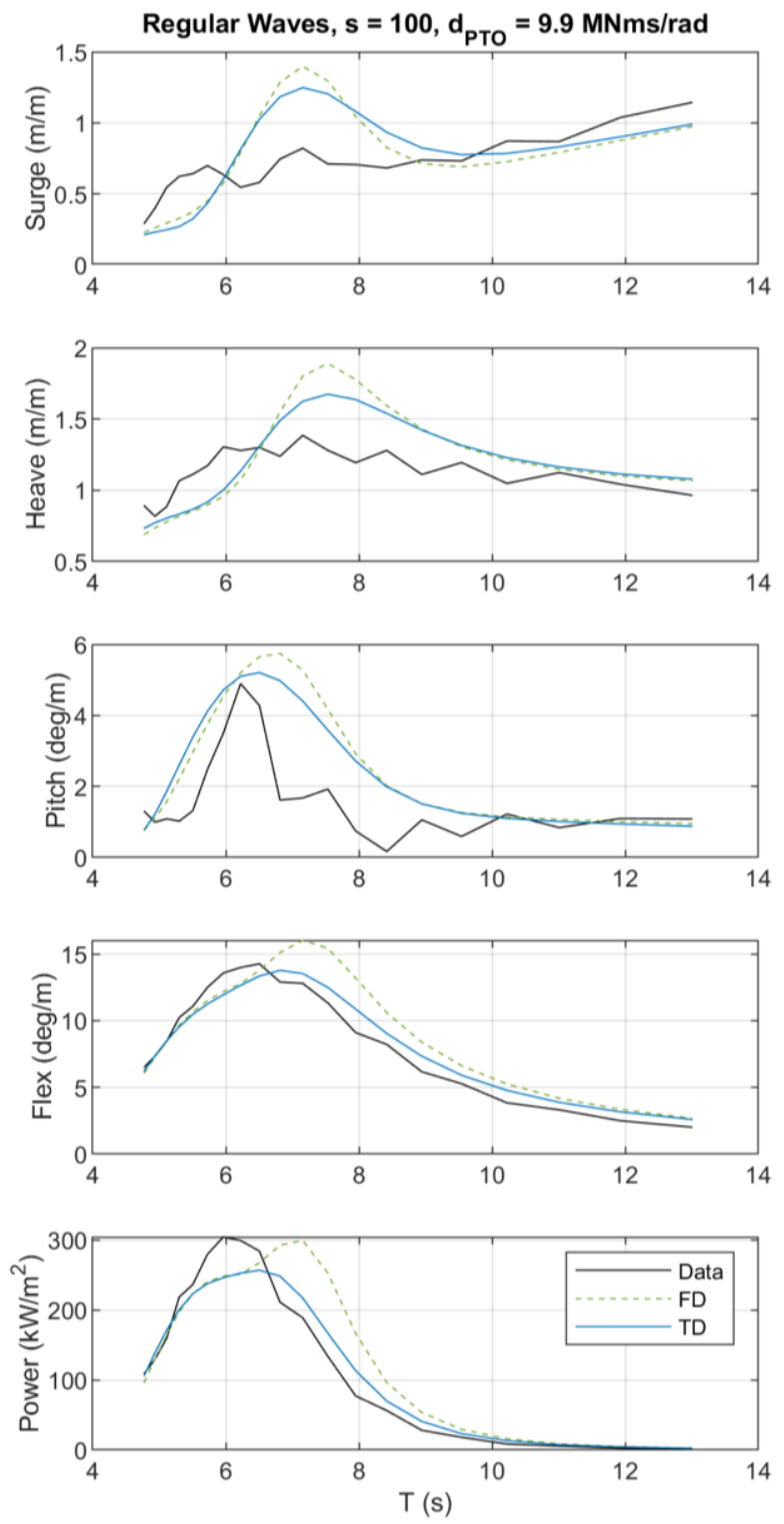

Fig. 9. RAO Test Set 2

$6.6 \mathrm{~s}$ than the higher at $9.6 \mathrm{~s}$. Nevertheless, the response in flex is still broad, and the peak RAOs in flex range between approximately $12-20 \mathrm{deg} / \mathrm{m}$ depending on the wave steepness and the PTO damping, which is still high compared to traditional hinged raft flex RAOs.

The agreement between the surge and heave responses is not as good as it was for pitch and flex. The data does not show peaks in surge and heave motions that are present in the numerical models.

The power RAO response follows the flex response but magnifies differences between results as it is proportional to the square of the flex response.

What is very interesting is that the data peak flex motion and correspondingly the peak of the power RAO exceeds that of the nonlinear numerical model and in some cases the linear model as well. This can be seen most clearly in Figs 8 and 9, which show the response at wave steepness of $s=100$ for two damping levels. In Fig 8 , the data power RAO exceed that on the time-domain model by $100 \mathrm{~kW} / \mathrm{m} 2$.
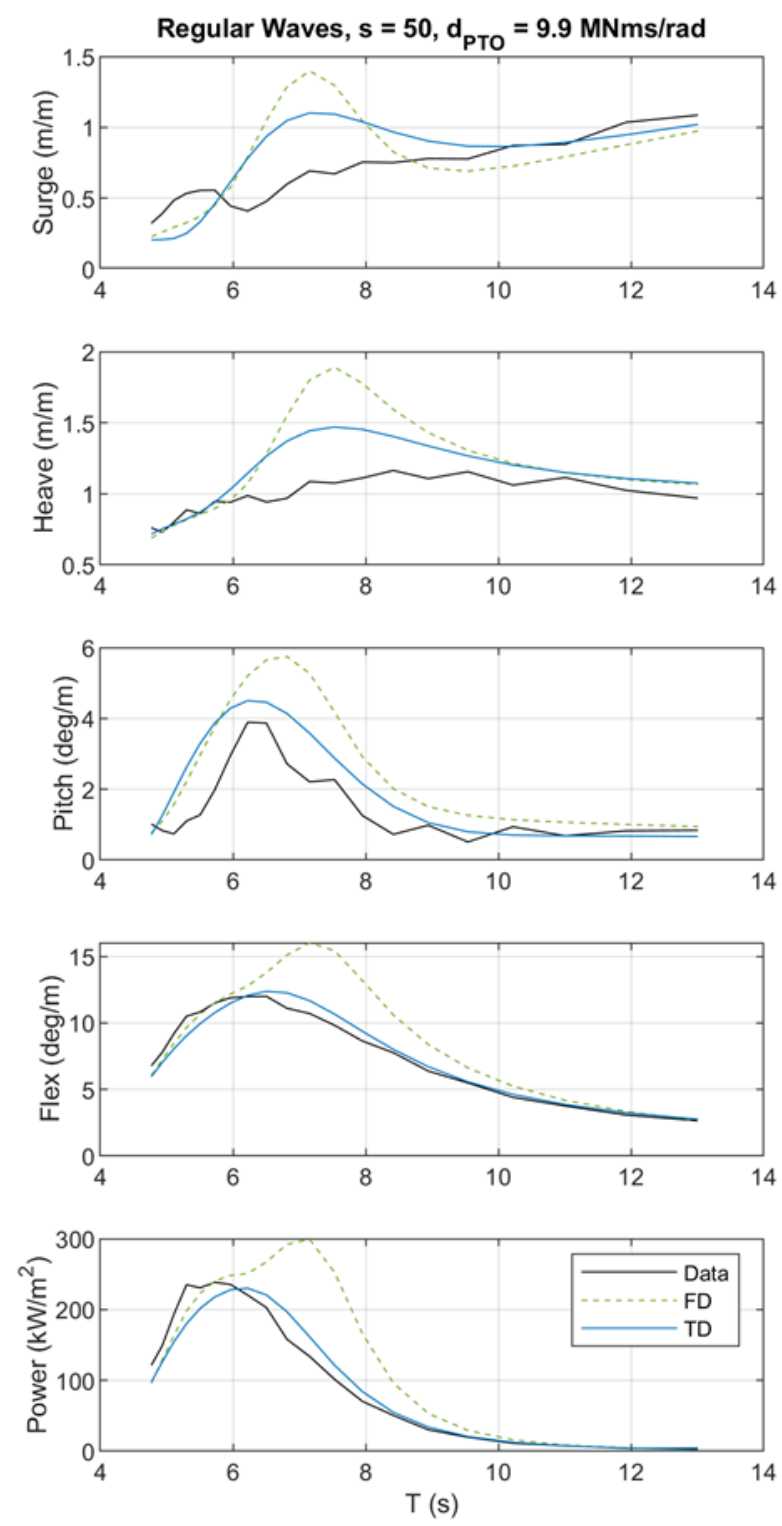

Fig. 10. RAO Test Set 3.

Also in all cases, the peak of the data power response is at a lower wave period than that of the numerical models. This can be seen most clearly in Fig. 9, where the linear model response shows a peak in the power at $7.2 \mathrm{~s}$, while the data has a peak at $6 \mathrm{~s}$.

That the data peak is higher and at a different frequency than the numerical models suggests two causes.

1) There is some significant linear force that is not being modelled correctly in the numerical models. Possibilities include an incorrect assessment of the mass-inertia due to water uptake in 3D printed part of the wave-tank model, an incorrect assessment of the tank model waterline leading to incorrect added mass value, or a poor calculation of the mooring stiffness. It should be noted that Mocean looked extensively into these possibilities and was not able to find anything conclusively.

2) The other possible reason for the difference is that there is a nonlinear force that is not being modeled in 


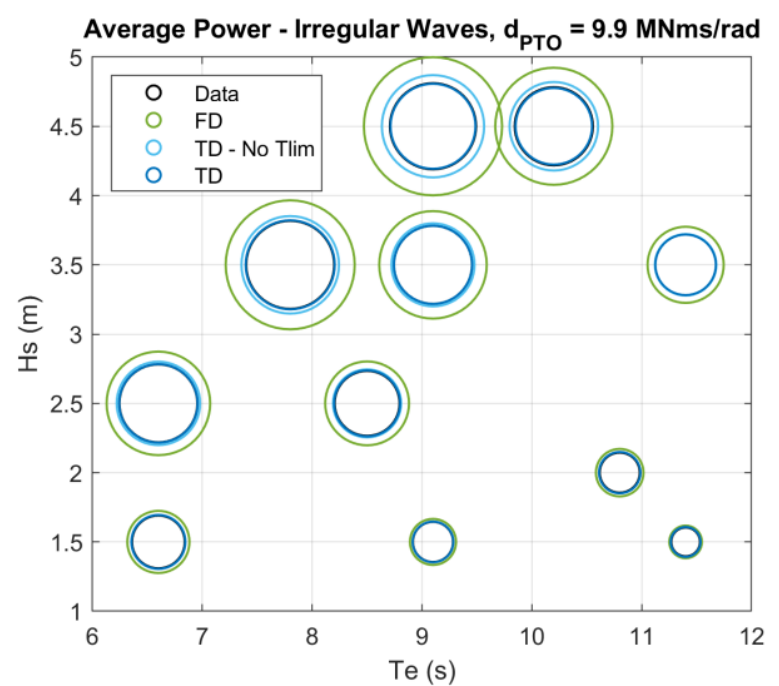

Fig. 11. Power plots Test Set 4 . Hs is significant wave height. Te is energy period.

either numerical model that acts to shift the wave period of and actually increase the peak of the response. The nature of this possible phenomenon is unknown but is of interest for its potential to enhance WEC performance.

A final worthwhile comparison is to consider the differences between the linear frequency-domain model and the nonlinear time-domain model. The nonlinear time-domain model shows a reduced response in power absorption compared to the linear, and except for the high data peak, is a better fit to the data than the linear model.

\section{G. Irregular wave response}

Figures 11 and 12 show the average power absorption in each sea state for the data, the linear frequency domain model, and the nonlinear time-domain model under two settings: without and with the torque limit applied. The torque limit, when applied, is the same as that modelled in the data. Average power absorption is given by the size of the circles - larger circles indicating more absorption.

In many cases the data circle which is in black cannot be seen because it is overlapped by the nonlinear torquelimit results (dark blue) which are in very good agreement.

The agreement between the nonlinear torque limit model and the data is very good, less than $10 \%$ error, for all sea states at both damping levels. This is notable because the sea states go to $\mathrm{Hs}=4.5 \mathrm{~m}$, which is a condition where one would expect nonlinear hydrodynamics due to wetted geometry changed to be significant. However, the power absorption is reasonably captured using only a nonlinear viscous Morison model and a nonlinear torque limit.

Results of the nonlinear model without the torque limit were included to show the proportionate impact of the nonlinear Morison force, which accounts for most of the difference between the linear and the nonlinear models; that is, the difference between the green (linear) and light

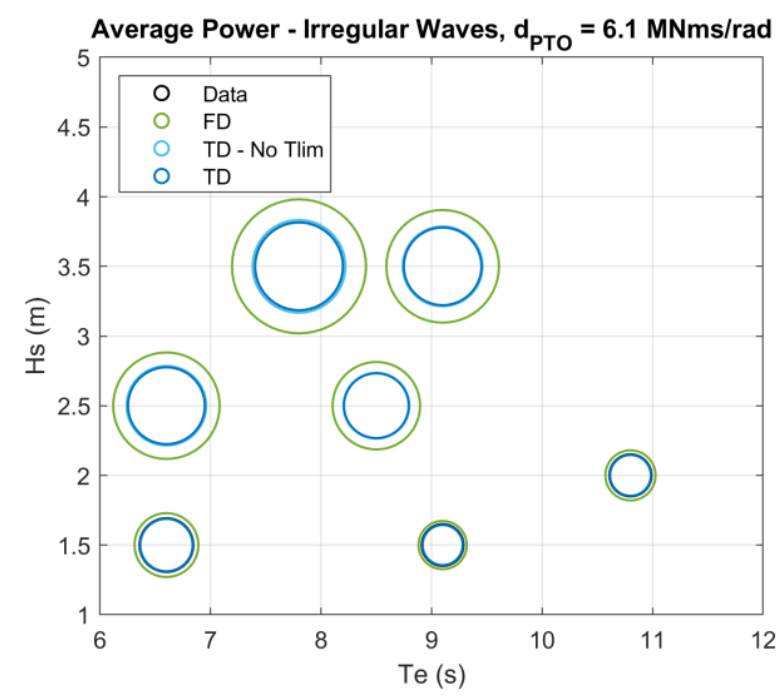

Fig. 12. Power plots Test Set 5. Hs is significant wave height. Te is energy period.

blue (nonlinear, no torque limit) is greater than the difference between the light blue and dark blue circles.

Unsurprisingly, the linear model over estimates the power absorption, and this overestimate gets significantly worse in high sea states. This shows the importance of including nonlinearities in numerical models for accurate power prediction.

\section{CONCLUSION}

Due to its geometry, the Mocean M100 WEC has an undamped response with two resonant frequencies and a broad bandwidth. The introduction of PTO damping and viscous damping eliminates the sharp resonant peaks, and the overall response is closer to the lower period peak. However, the bandwidth is still broad, and the flex RAO shows peaks of approximately $12-20 \mathrm{deg} / \mathrm{m}$ depending on the PTO damping and wave steepness.

The agreement between the numerical models and data for regular waves is reasonable in pitch and flex, but the numerical model over predicts the response in surge and heave.

Very interestingly, in flex, and correspondingly in power, the peak of the data RAO exceeds that of the numerical models. This suggests that either some linear force is not modelled correctly or that there is a yet-to-be known nonlinear force that enhances the response. If it is a nonlinear force, it has the potential to be beneficially exploited for wave energy absorption.

Despite some disagreement in the RAOs, in terms of average power, there is good agreement between the data and the nonlinear time-domain model when including the quadratic Morison force and the nonlinear torque limit. This agreement extends up to $\mathrm{Hs}=5 \mathrm{~m}$.

Comparisons between the linear and nonlinear models show that the linear model significantly over predicts the power at high wave heights and shows the importance of using nonlinear forces. Interestingly, the nonlinear forces used in this case were quite simple and fast to compute.

In conclusion, results from numerical and physical models show the benefit of asymmetric WEC geometry 
for a hinge raft: force coupling between modes of motion enables better frequency tuning and broader bandwidth of the WEC response in the PTO DOF. This allows improvements in the ratios of power absorption to body size and power absorption to maximum torque.

\section{ACKNOWLEDGEMENT}

This report is work commissioned by Wave Energy Scotland. The views expressed in this publication are those of the author(s) and not necessarily those of Wave Energy Scotland.

\section{REFERENCES}

[1] Waveenergyscotland.co.uk, "About Us", 2019. [Online]. Available: http://www.waveenergyscotland.co.uk/. [Accessed: 08-Jan-2019].

[2] Apparatus for extracting energy from wave movement of the sea, by Christopher Cockerell. (1978, July 4). US4098084A [Online]. Available: https://patents.google.com/patent/US4098084.

[3] Spaced apart wave generator float array, by Glenn E. Hagen. (1983, July 12). US4392349A [Online]. Available: https://patents.google.com/patent/US4392349A.

[4] WAMIT, Inc.., "WAMIT User Manual Version 7.2," 2016.

[5] M. Folley and T. Whittaker, "Spectral modelling of wave energy converters," Coastal Engineering, vol. 57, pp. 892-897, 2010.

[6] M. Ó'Catháin, B. J. Leira, J. V. Ringwood, and J.-C. Gilloteaux, "A modelling methodology for multibody systems with application to wave-energy devices," Ocean Engineering, vol. 3513, pp. 1381-1387, 2008.

[7] D. Padeletti, R. Costello, J. V. Ringwood, "A multi-body algorithm for wave energy converters employing nonlinear joint representation," in Proc. of the ASME 2014 33rd International Conference on Ocean, Offshore, and Arctic Engineering, San Francisco, USA, 2014.

[8] Flowavett.co.uk, “Tank Design and Features, 2019. [Online]. Available: https://www.flowavett.co.uk/tank-design-andfeatures. [Accessed: 24-April-2019]. 